\title{
MODELING STENT EXPANSION DYNAMICS AND BLOOD FLOW PATTERNS IN A STENOTIC ARTERY
}

\author{
M.R. HYRE ${ }^{1}$, R.M. PULLIAM ${ }^{2}$ \& J.C. SQUIRE ${ }^{1}$ \\ ${ }^{1}$ Department of Mechanical Engineering, Virginia Military Institute, USA. \\ ${ }^{2}$ Department of Mechanical Engineering, Villanova University, USA.
}

\begin{abstract}
Restenosis remains a significant problem in coronary intervention. Although stent migrations, collapses, and positioning difficulties remain serious issues, it is the problem of restenosis which is the most common long term problem in treating atherosclerotic coronary arteries with stents. Although much attention has focused on biocompatibility, thrombosis and neointimal pathology, less attention has been given to matching stents to the inflation balloon, artery and occlusion size. Balloons are typically sized 1-2 mm longer than endovascular stents, yet the effects of the degree of balloon overhang are unknown. In this study, a computational model capable of predicting balloon/stent/artery interactions and their effects on arterial stresses was developed to assess the effects of length mismatch on stent expansion characteristics and arterial stresses. Results from this study indicate that maximum arterial stress at balloon contact is approximately proportional to the degree of balloon overhang. A $100 \%$ increase in balloon overhang results in a $4 \%$ increase in maximum endflare and a $39 \%$ change in the peak arterial stress. However, at the end of expansion, which is of the most clinical importance, the increase in maximum endflare is $2 \%$ and the increase in maximum arterial stress is $93 \%$ at the balloon point of contact and $45 \%$ at the point of contact with the far proximal and distal ends of the stent. When comparing the results of calcified and cellular plaque, a maximum endflare of about $55 \%$ was observed for both the calcified and cellular plaque cases during expansion. At the end of expansion the increase in maximum endflare was $10 \%$ for the cellular plaque and $40 \%$ of the calcified plaque. The peak equivalent stress seen by the artery was about $100 \%$ larger in the cellular case than in the calcified plaque case.
\end{abstract}

Keywords: balloon inflation, finite element modeling, stenosis, stent inflation.

\section{INTRODUCTION}

Atherosclerotic stenosis and its ischemic complications necessitate arterial reconstruction. Current strategies to restore normal blood flow in stenotic coronary arteries include angioplasty, intracoronary stents, and coronary artery bypass surgery. Depending on the method of treatment, the incidence of restenosis is high: up to $40 \%$ within 6 months after angioplasty [1], 25\% after stenting [2], and $20 \%$ after bypass surgery [3]. Therefore, restenosis remains a significant problem in coronary intervention.

Advances in prosthetic science and engineering have spurred the rapid development of many new permanent implants such as arterial reinforcement grafts, venous filters, myocardial perforationsealing clamshells, and stents that strengthen and scaffold the biliary duct, urethra, veins, and arteries. These devices are typically attached to a delivery catheter and threaded to the site of interest where they are expanded. The very nature of the remote delivery systems make the mechanical details of implantation difficult to ascertain, yet this is important to quantify since there may be a link between how the devices are emplaced and the body's acute and chronic response. Endovascular stents in particular are ideal devices to quantify these relationships because of the extreme levels of stress they impose and because of their ubiquity; more than one million are annually implanted in the U.S. alone [4].

These studies suggest an upper limit exists to the success of purely biomedical approaches for managing post-device implantation, and a return to examining the mechanical initiators of vascular injury that occur during implantation. A complete understanding of the manner that stents expand 
may thus lead to both a new understanding of the processes of vascular adaptation to implants and possibly to the design and development of less-injurious devices. Experimental data are indirect; stents are too thin to be fully radio-opaque, and methods of bringing a camera to the stent, such as intravascular ultrasound, are blocked by the balloon that expands the stent during the critical moments of implantation. Postmortem examinations indicate that restenosis is paradoxically more severe in the parts of the artery immediately outside the stented region, and animal studies have shown an unusual pattern of endothelial cell denudation occurring at a regular pattern at the center of stent struts, a superficial injury that may be a marker for deep vascular injury [5]. These data are not explained by current finite element analyses of arterial stresses in a stent-expanded artery [6-9] because no finite element models included the expansion of a balloon catheter in the model of a plastically deformed stent.

The inclusion of the balloon catheter in the stent expansion model is not trivial. The problem is highly nonlinear and includes complex contact problems among the stent, balloon, and arterial wall. Additionally, the balloon properties change dramatically depending on whether it is fully or partially inflated.

\section{GEOMETRY}

The finite element stress analysis was performed on a three-dimensional stent/balloon/plaque/artery geometry. In addition to the usual difficulties in modeling the mechanical behavior of soft tissue, the overall system response is highly nonlinear due to the large plastic/multilinear-elastic/hyperelastic deformations of the individual components. The component geometries and constitutive material models are described below.

\subsection{Artery}

The coronary artery model was $30 \mathrm{~mm}$ in length, with an inside diameter of $2.8 \mathrm{~mm}$ and thickness of $0.3 \mathrm{~mm}$. Average element size was about $0.5 \mathrm{~mm}$ long with a thickness of $0.15 \mathrm{~mm}$. This configuration yielded a total of 7,680 elements. The artery elements were defined by eight nodes capable of large deflections and hyperelasticity.

\subsection{Plaque}

The plaque has a semi-parabolic profile and corresponds to percent blockage data presented by Lally et al. [9]. The plaque was $16 \mathrm{~mm}$ in length with a maximum thickness of $0.48 \mathrm{~mm}$. This configuration corresponds to a maximum percent blockage of about $60 \%$. The characteristic shape of the plaque can be seen in Fig. 1.

\subsection{Balloon}

The balloon was modeled in its unfolded state, and already assumed to be in contact with the stent. The balloon dimensions are given at $0 \mathrm{~Pa}$, before stent expansion occurs. Depending on the amount of balloon overhang, the overall length of the balloon can range from 22 to $23 \mathrm{~mm}$. For $1 \mathrm{~mm}$ balloon overhang, the total length of end balloon is $1 \mathrm{~mm}$, or $0.5 \mathrm{~mm}$ on each side of the balloon, yielding a total balloon length of $22 \mathrm{~mm}$. For $2 \mathrm{~mm}$ balloon overhang, the total length of balloon overhang is 2 $\mathrm{mm}$, or $1 \mathrm{~mm}$ on each side, yielding a total balloon length of $23 \mathrm{~mm}$ (Fig. 2).

The balloon was meshed using triangular shell elements with an average base size of $0.05 \mathrm{~mm}$ and an average side length of $0.05 \mathrm{~mm}$. This yielded 54,456 elements for the 2-mm overhang case and 


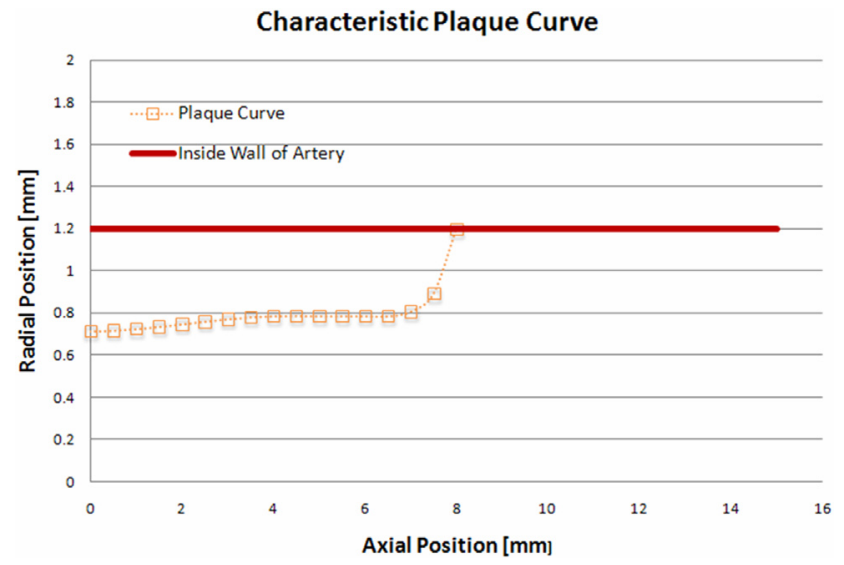

Figure 1: Characteristic plaque curve.

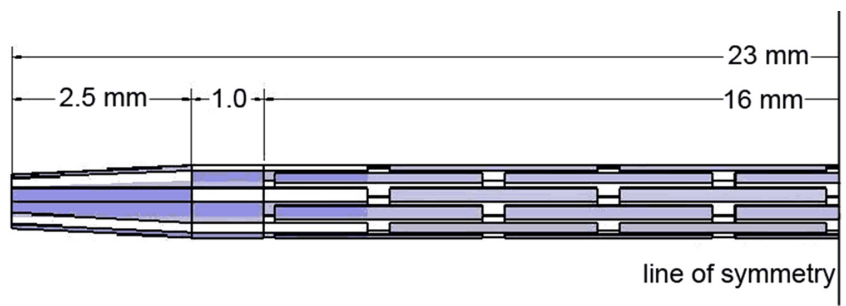

Figure 2: Balloon geometry shown with mounted slotted tube stent.

51,616 elements for the 1-mm overhang case. For finite element analysis, elements capable of modeling shell structures, large deflections and plasticity were used.

\subsection{Stent}

A three-dimensional model of the slotted tube geometry intravascular stent was created. The stent is $16 \mathrm{~mm}$ in length (L), with an inside diameter (ID) of $1 \mathrm{~mm}$, and a thickness (t) of $0.1 \mathrm{~mm}$. The diamond-shaped stent consists of 5 slots in the longitudinal direction and 12 slots in the circumferential direction with a length of $2.88 \mathrm{~mm}$. The slots were cut such that in a cross-section, the angle describing the slot was approximately 23 degrees, and the angle describing the metal between slots was 6.9 degrees (Figs 3 and 4). These dimensions refer to the model in an unexpanded state $[7,9]$.

The stent was meshed using hexahedral elements. There are two elements through the thickness of the stent yielding a total of 12,036 elements. The stent was assigned an element type solid45 for analysis in ANSYS. These elements are defined by eight nodes and capable of large deflections and plasticity.

The stent and balloon mesh is shown in Fig. 5. A cross-section of the final model geometry with the stent/balloon/artery and plaque is shown in Fig. 6. 


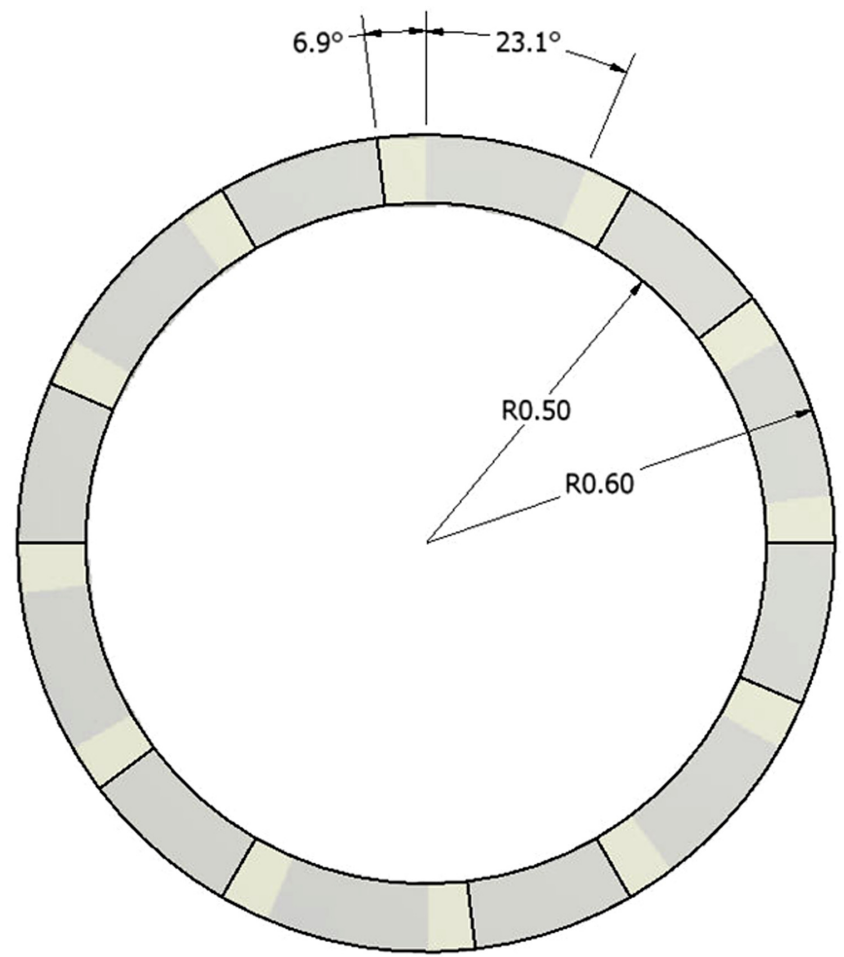

Figure 3: Medial slice of modeled slotted tube stent.

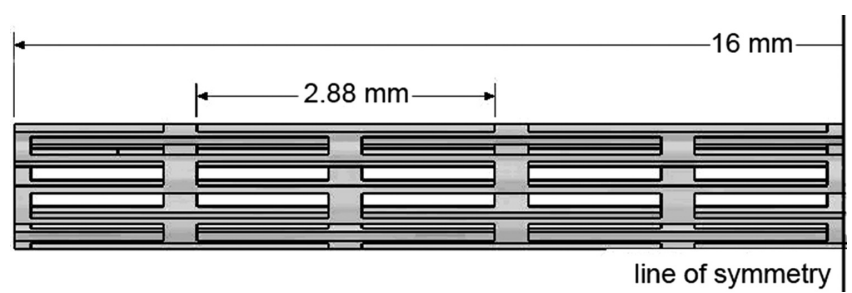

Figure 4: Side view of stent geometry.

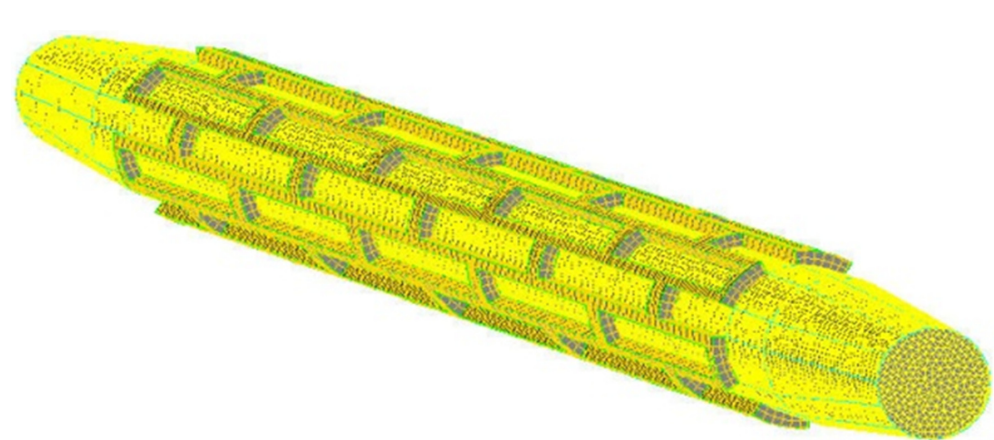

Figure 5: Balloon/stent mesh. 


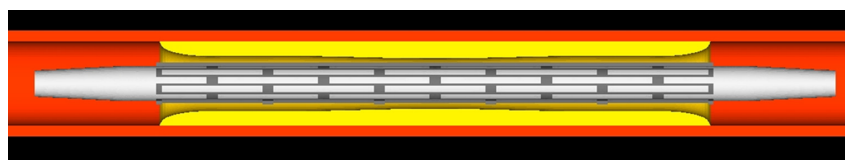

Figure 6: Final model geometry.

\section{MATERIALS}

\subsection{Artery}

The material properties of the artery are based on a previous study by Lally et al. [6]. This model describes the behavior of the artery using a five-parameter, third-order, Mooney-Rivlin hyperelastic constitutive equation. This model has been found to be suitable for modeling an incompressible isotropic material [10].

Lally et al. developed the constants for this model by fitting the five-parameter Mooney-Rivlin expression to uniaxial and equibiaxial tension tests of human femoral arterial tissue data [11] (see Table 1).

The material properties of the plaque are based on a previous study by Loree et al. [12]. Two histological classifications of plaques were modeled: cellular and calcified. The cellular and calcified specimen results were chosen to provide models of stent expansion dynamics with plaques whose stress-strain slopes differed significantly. This model describes the behavior of the plaque using a five-parameter, third-order Mooney-Rivlin hyperelastic constitutive equation. This model for plaque behavior neglects the artery laminate compositions, tissue anisotropy as well as the residual strain and active smooth muscle stresses [13]. The final form of the strain density function used to model the artery is given in eqn (1). The constants were developed for this model by fitting the five-parameter Mooney-Rivlin expression to uniaxial tension tests of human aortic atherosclerotic tissue data [12]. The hyperelastic constants for the plaques are given in Table 2.

\subsection{Balloon}

To model the mechanical properties of the balloon without evaluating the balloon's behavior during unfolding, empirical data were used (Fig. 7). The stress-strain curve for the full expansion of the balloon produced a linear piecewise function. The first segment of the piecewise function is representative of the unfolding balloon, whereas the second is of the balloon expansion after unfolding.

Table 1: Hyperelastic constants for artery.

\begin{tabular}{l} 
Hyperelastic constants $[\mathrm{Pa}]$ \\
\hline$a_{10}=0.01890$ \\
$a_{01}=0.00275$ \\
$a_{20}=0.08572$ \\
$a_{11}=0.59043$ \\
$a_{02}=0$
\end{tabular}


Table 2: Hyperelastic constants for plaque.

\begin{tabular}{ll}
\hline \multicolumn{2}{c}{ Hyperelastic constants [Pa] } \\
\hline Cellular plaque & Calcified plaque \\
\hline$a_{10}=-0.088314$ & $a_{10}=-3.0254$ \\
$a_{01}=0.10619$ & $a_{01}=3.1073$ \\
$a_{20}=0.11373$ & $a_{20}=107.39$ \\
$a_{11}=0.89382$ & $a_{11}=-234.7$ \\
$a_{02}=-0.96676$ & $a_{02}=137.22$ \\
\hline
\end{tabular}

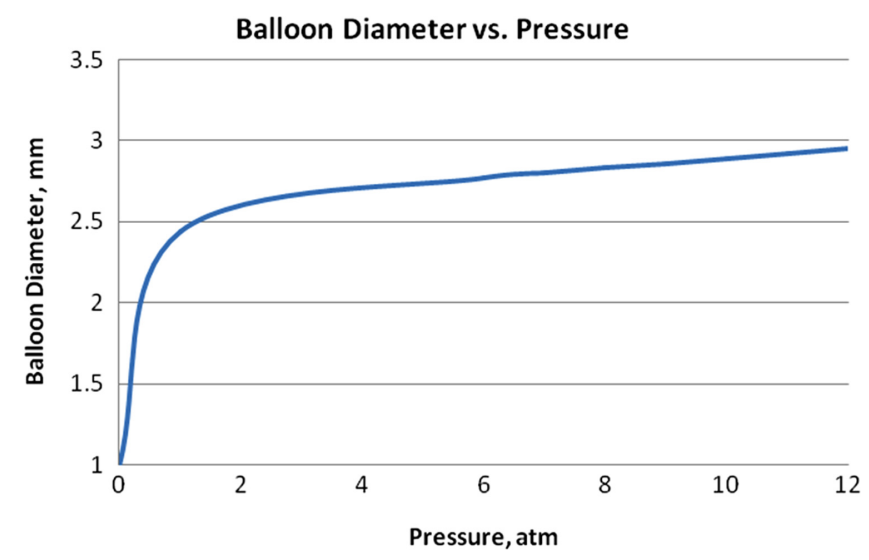

Figure 7: Experimentally determined balloon inflation curve.

\subsection{Stent}

The stent was modeled after the slotted tube geometry given by Migliavacca et al. [7]. This model assumes the stent to be made of $316 \mathrm{LN}$ stainless steel. The Poisson ratio is 0.3 and the Young Modulus is $200 \mathrm{GPa}$. The plastic region of the stress-strain curve is shown in Fig. 8.

\section{BOUNDARY CONDITIONS}

The artery, balloon, plaque, and stent were all constrained in the rotational directions allowing no rotation. The artery was constrained axially at the distal ends. The artery was at a minimum $7 \mathrm{~mm}$ longer than the stent on each side and $3.5 \mathrm{~mm}$ longer than the end of the balloon on each side. This constraint on the artery did not affect the behavior of the artery at the point of contact with the stent or balloon because of the extra length of the artery on both sides. The same axial constraint was placed on the balloon. To model the expansion of the balloon, the balloon was assigned a ramped internal pressure load.

\section{DEFORMED GEOMETRY EXPORT AND SOLUTION}

In order to examine the blood flow patterns through the stented artery, a script was written to export the final expanded stent/plaque/artery geometry for meshing. This mesh was then used within a finite volume computational fluid dynamics code (FLUENT/UNS) to predict arterial wall shear 


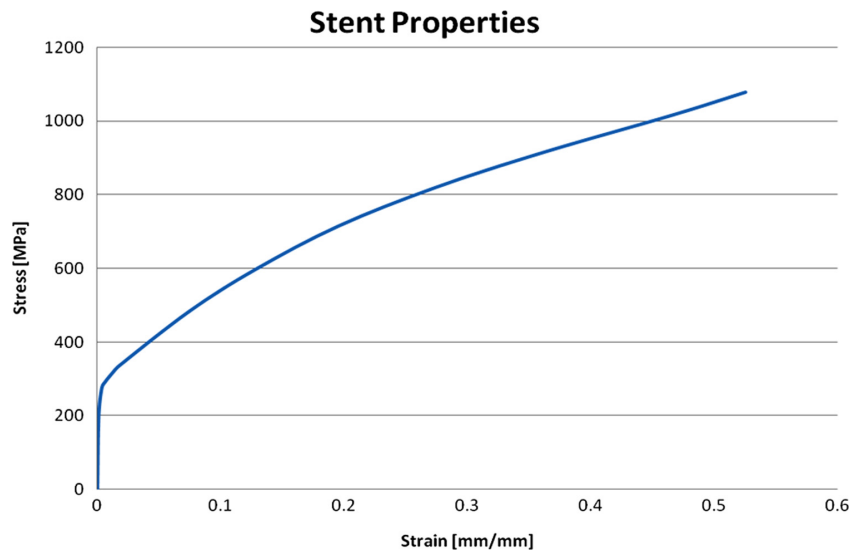

Figure 8: Stent stress-strain curve.

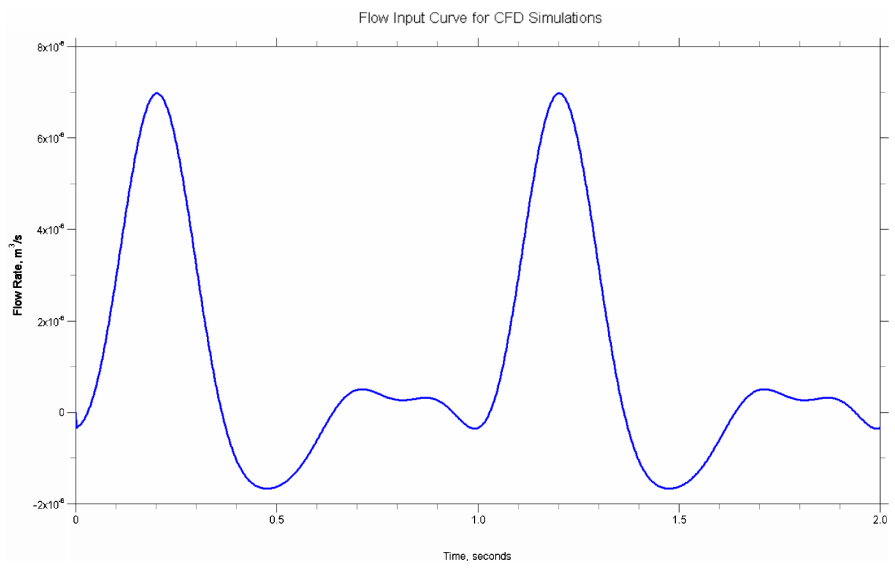

Figure 9: Flow inlet boundary condition for CFD simulations.

stresses and blood velocity and strain rate distributions. The deformed geometry was exported to STL format and the internal geometry (solid mirror of the stent/plaque/artery) was meshed using a hex dominant grid. Holes and seams between the various geometry parts were automatically filled within the meshing algorithms.

The inlet boundary condition was specified as a velocity inlet using the time dependent flow rate equation of Womersley [14] (Fig. 9). The flow was assumed to enter the simulation with a constant cross-sectional velocity. A no-slip condition where the stent/plaque/artery surfaces were in contact with the blood was assumed. The Casson model was used to evaluate the viscosity of the blood since the artery was relatively large and inhomogeneities associated with Fahraeus effects were negligible.

\section{RESULTS AND DISCUSSION}

Figure 10 shows the endflare during stent expansion for the $1 \mathrm{~mm}$ and $2 \mathrm{~mm}$ balloon overhang cases when there is no plaque included in the models. Endflare is defined as the ratio of the stent diameter at the distal ends to the diameter at the stent centerline. Figure 11 shows the condition of the stent/ 


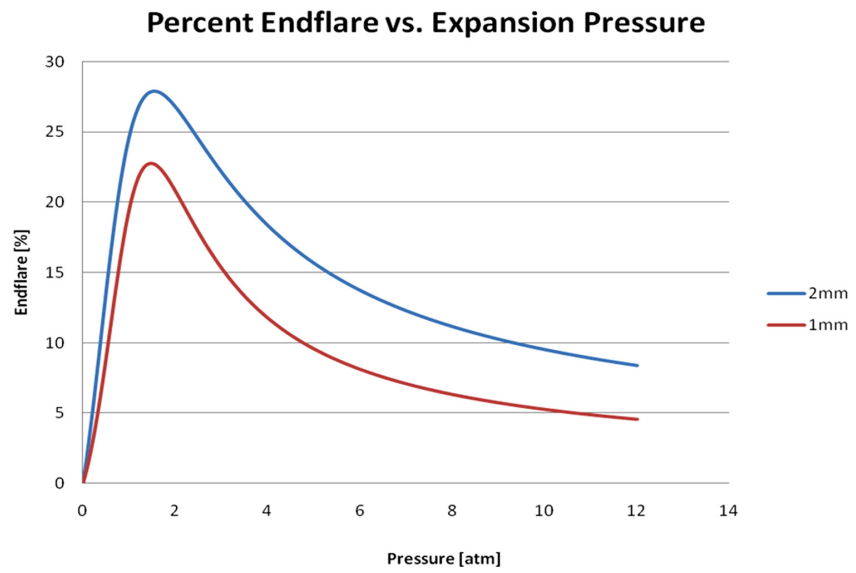

Figure 10: Endflare without plaque for $2 \mathrm{~mm}$ (top curve) and $1 \mathrm{~mm}$ (bottom curve) overhang.

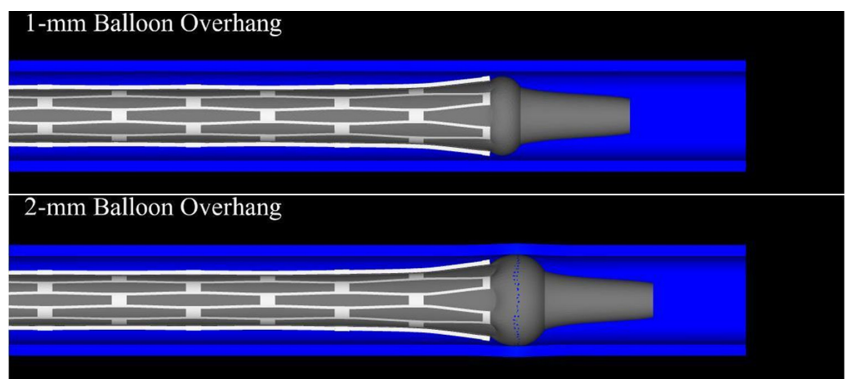

Figure 11: Stent/balloon/artery system at the point of maximum endflare.

balloon/artery system at the point of maximum endflare. There is a significant difference in the endflare both at the point of peak endflare and at the end of expansion, indicating that the amount of balloon overhang can have a significant impact on vascular injury.

Figure 12 shows the amount of balloon/artery interaction during stent inflation. As one would expect, there is a significant increase in the area of balloon/artery contact of approximately $33 \%$.

Figure 13 shows the endflare during stent expansion for the $2 \mathrm{~mm}$ balloon overhang case when calcified and cellular plaques are included in the models. The endflare with plaque present in the model is significantly higher than when it was not included. This is because the distal ends of the stent were located such that they did not contact the plaque. Therefore, the effective diameter against which the stent was expanding was significantly larger in this area leading to a lower expansion resistance. There is a significant difference in the endflare both at the point of peak endflare and at the end of expansion, indicating that the amount of balloon overhang can have a significant impact on vascular injury.

Figure 14 shows the arterial stresses at the end of stent expansion when calcified and cellular plaque was included in the model. At the end of expansion the increase in maximum endflare for the cellular plaque geometry over the calcified plaque geometry is about $200 \%$. The increase in maximum arterial stress is $200 \%$ at the point of stent contact at the proximal and distal ends. Figure 15 shows the max arterial stress during the expansion process for the cellular and calcified plaque cases. 


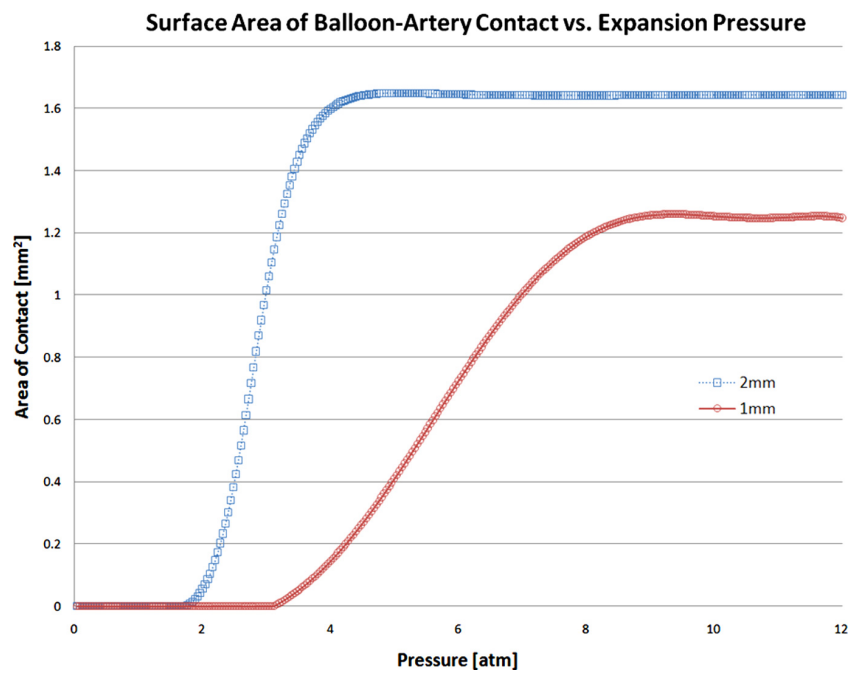

Figure 12: Comparison of balloon/artery contact areas.

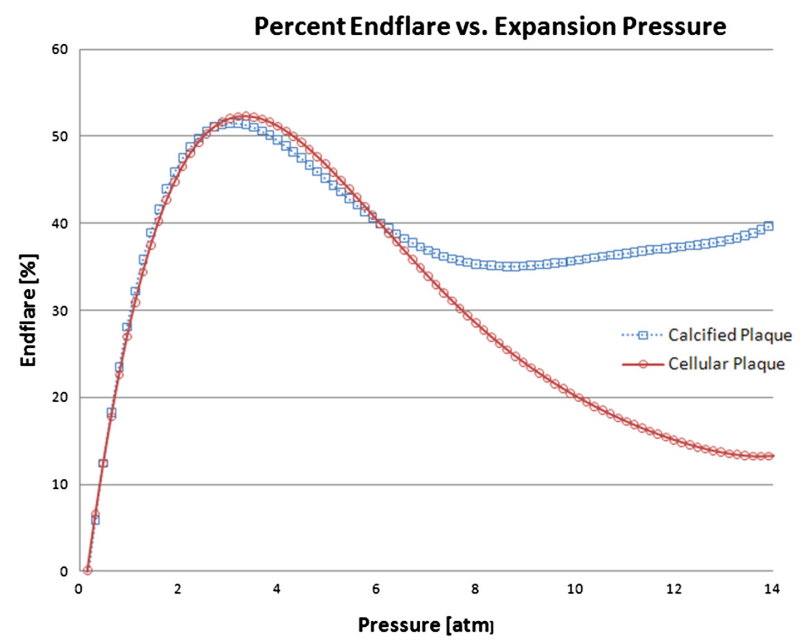

Figure 13: Endflare with plaque for calcified (blue) and cellular (red) plaque.

The cellular plaque case results in much higher stresses at a given balloon expansion pressure than the calcified plaque case.

Figures 16 and 17 show the directional stresses when calcified and cellular plaque are included in the model. As expected, the arterial stresses were significantly lower for the calcified plaque cases when compared to the cellular case. This is the result of the much higher rigidity of the cacified plaque layer over the celluar plaque. This is further supported by the higher stresses seen by the plaque in the calcified case as compared to the cellular case.

It should be mentioned that no plaque breakup model was included in the simulations. It would be expected that the calcified plaque would break up before the high stresses at the end of expansion predicted by the model. 


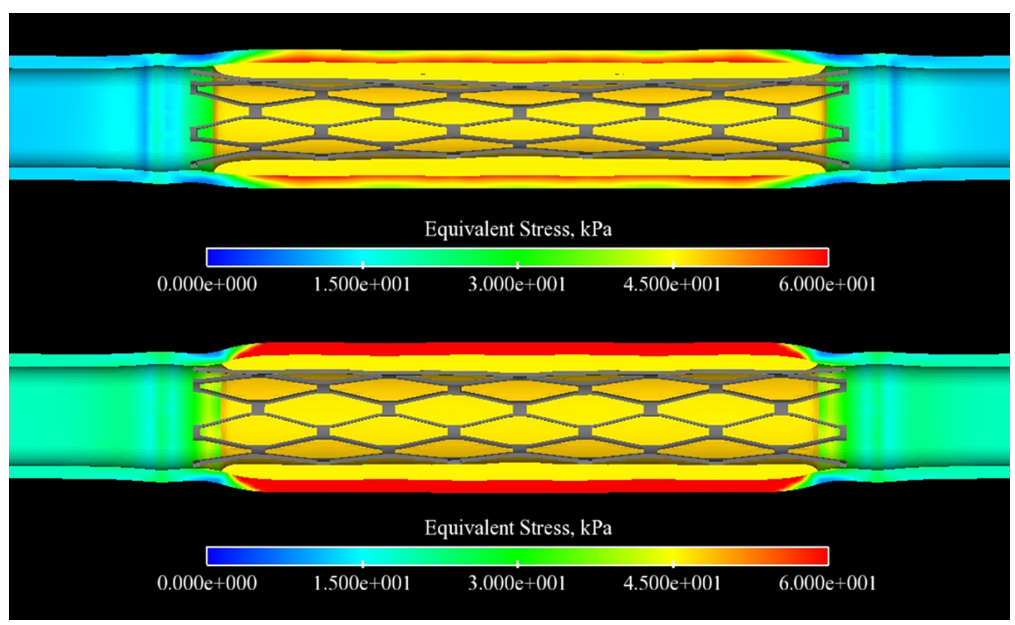

Figure 14: Arterial/plaque stresses for calcified (top) and cellular plaque (bottom).

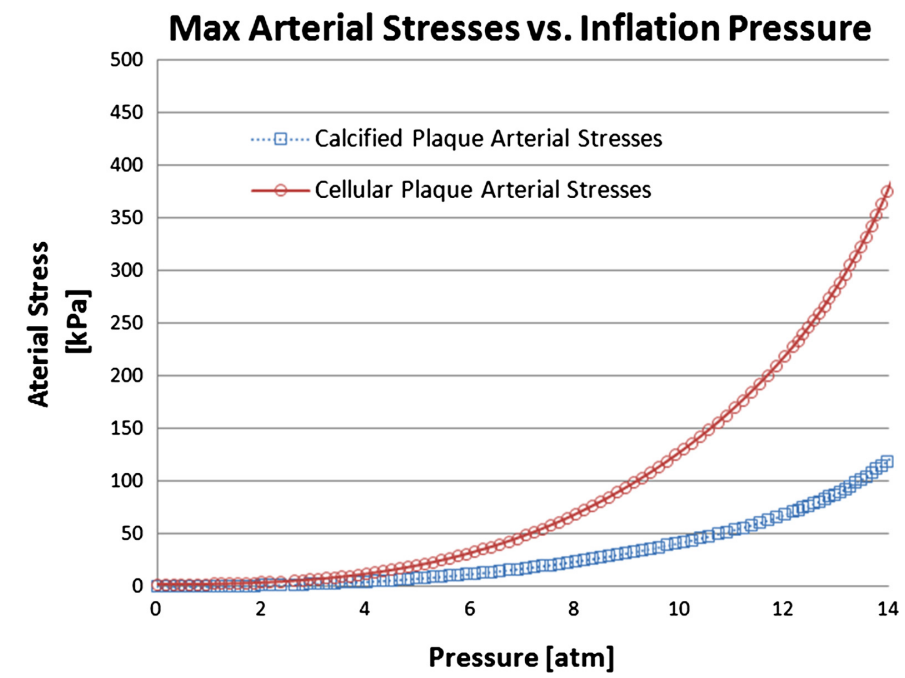

Figure 15: Max arterial stresses for calcified and cellular plaque.

As one would expect, the most significant compenent of stress is the hoop. The smallest compenent is the axial. The clinical significance of the directional stresses on vascular injury is unclear. Future experimental studies will be aimed at determining the most appropriate stress to analyze when evaluating vascular injury (von Misses, maximum principal, directional, etc.). The current model does not include the anisotropic behavior of the artery and plaque materials. Additionally, no distinction was made between the passive arterial medium and the active fibers, the orientations of which may vary from inner to the outer layers of the arterial wall. This is currently being included in a more sophisticated model which also includes arterial prestresses.

Finally, Fig. 18 shows the preliminary results of the flow pattern within the final stented geometry. The development of Poiseuille flow is apparent as the blood moves from the model entrance along 


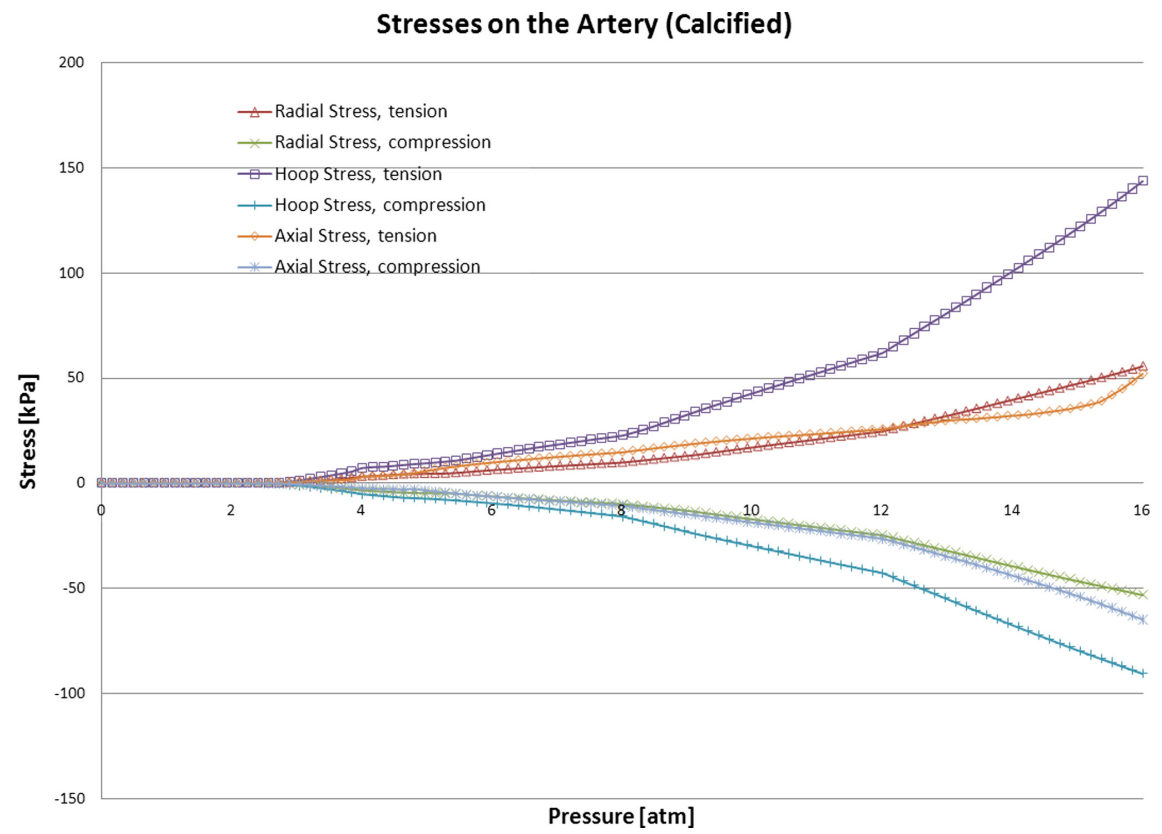

Figure 16: Arterial stresses (radial, hoop, and axial) for calcified plaque.

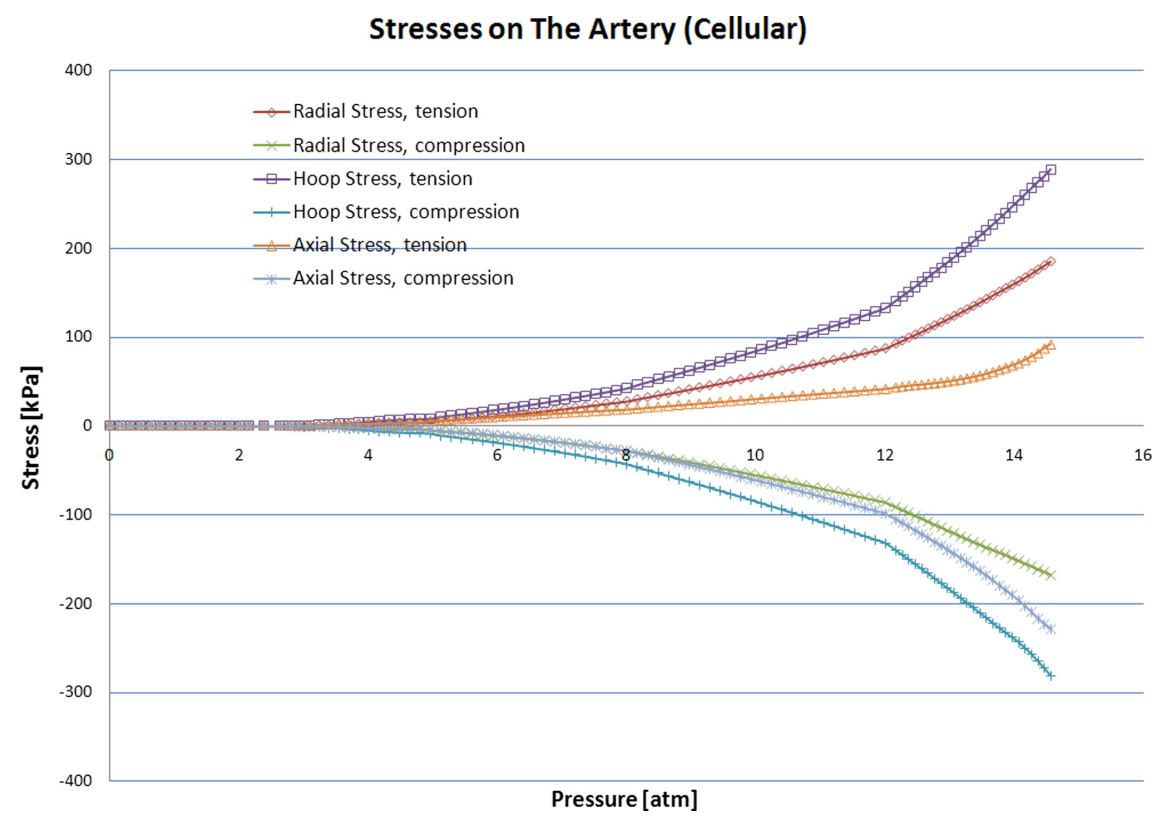

Figure 17: Arterial stresses (radial, hoop, and axial) for cellular plaque. 


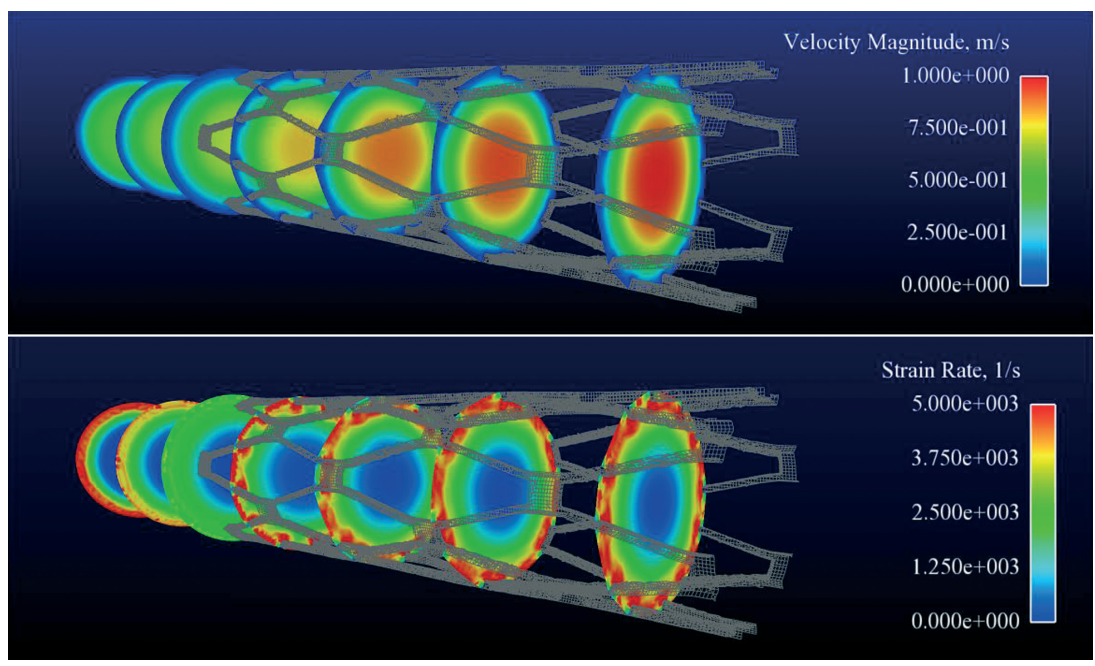

Figure 18: Velocity magnitudes and strain rates in stented artery.

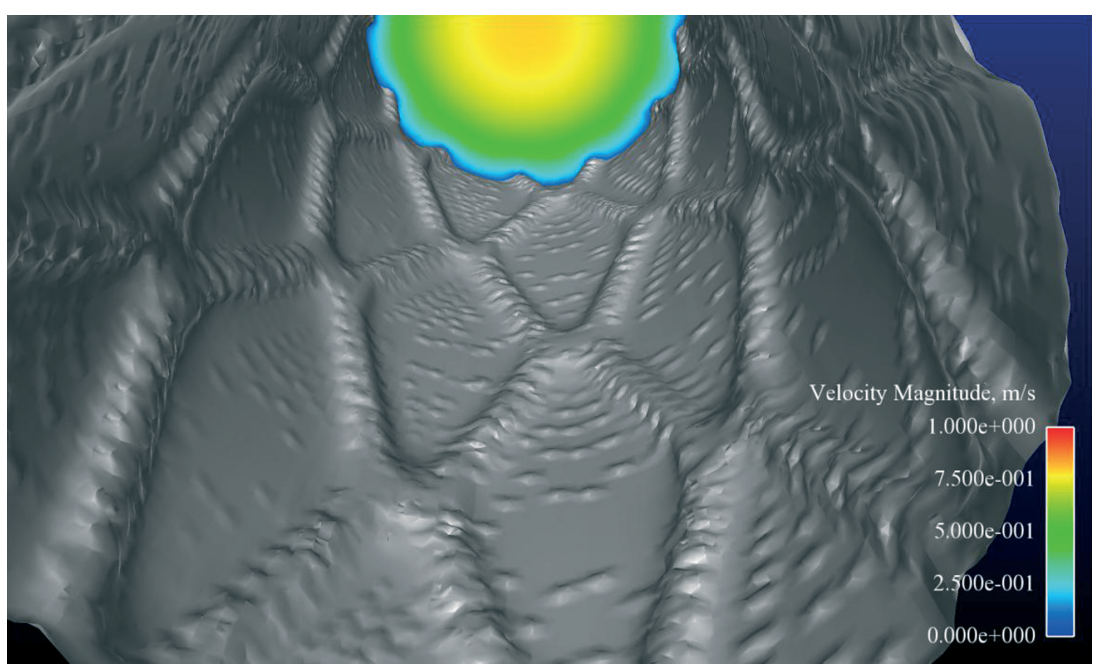

Figure 19: Iso-contour at $0.05 \mathrm{~m} / \mathrm{s}$ on region near plaque/stent interface.

the artery and plaque surface (not shown) and through the stented portion of the artery. The strain rates are quite interesting and show a decrease after the initial entry into the arterial section. However, they rapidly increase as the blood travels through the stented portion of the artery.

This CFD model is now being used to evaluate the diffusion process associated with drug eluting stents, as well as modeling neointima formation, thrombus formation mechanics, and blood flow patterns. It has been found that standing vortices and regions of stagnation are responsible for the rise in concentrations of platelet-activating agents within those regions. Platelets accumulate preferentially in the regions of large platelet-activating agent concentrations and low fluid velocities. Figure 19 shows a contour plot at $0.05 \mathrm{~m} / \mathrm{s}$. Regions of the domain near where the stent contacts the 
plaque exhibit zones of low velocity. These regions are potential locations for thrombus formation. Additionally, the wall shear rates and blood strain rates at the wall can be evaluated to determine if they exceed a critical embolizing limit.

\section{CLINICAL SIGNIFICANCE OF REPORT}

Concerns that drug-eluting stents interfere with the process of re-endothelization and thus may encourage long-term thrombosis have spurred interest in understanding the mechanisms causing acute de-endothelization during the stenting procedure $[15,16]$. This model aids in the prediction of regions of endothelial cell (EC) denudation during stent implantation. This is an important phenomenon since regions of EC denudation profoundly impact drug absorption/loading profiles of anti-proliferative agents in drug-eluting stents (DES). Additionally, anti-proliferative drugs are hypothesized to inhibit EC regrowth causing increased rates of long-term thrombosis, so predictive capability of regions of EC denudation during implantation provides the tool to reduce thrombosis rates of DES.

The model developed also helps in the prediction of regions of high arterial stresses which may cause vascular injury. Acute superficial and deep vascular injury has been found to be a strong predictor of chronic restenosis. This method provides a predictive tool to evaluate the degree of acute vascular injury of new stent geometries prior to in vivo studies.

Finally, the ability to examine blood flow patterns in stented arteries allows for the prediction of standing vortices and high platelet-activating substances capable of trapping and stimulating platelets for aggregation. It also allows for the evaluation of embolizing stresses acting on a thrombus.

\section{REFERENCES}

[1] Schillinger, M., Exner, M., Mlekusch, W., Haumer, M., Sabeti, S., Ahmadi, R., Schwarzinger, I., Wagner, O. \& Minar, E., Restenosis after femoropopliteal PTA and elective stent implantation: predictive value of monocyte counts. Journal of Endovascular Theory, 10, pp. 557-565, 2003. doi:10.1583/1545-1550(2003)010<0557:RAFPAE $>2.0 . C O ; 2$

[2] Antoniucci, D., Valenti, R., Santoro, G., Bolognese, L., Trapani, M., Cerisano, G. \& Fazzini, P., Restenosis after coronary stenting in current clinical practice. American Heart Journal, 135(3), pp. 510-518, 1998. doi:10.1016/S0002-8703(98)70329-1

[3] Griffiths, H., Bakhai, A., West, D., Petrou, M., De Souza, T., Moat, N., Pepper, J. \& Di Mario, C., Feasibility and cost of treatment with drug eluting stents of surgical candidates with multi-vessel coronary disease. European Journal of Cardiothroacic Surgery, 26, pp. 528-534, 2004. doi:10.1016/j.ejcts.2004.05.018

[4] Feder, B.J., Panel urges caution on coated stents. New York Times - Health p. 1, 2006.

[5] Rogers, C., Parikh, S., Seifert, P. \& Edelman, E.R., Endogenous cell seeding: remnant endothelium after stenting enhances vascular repair. Circulation, 11, pp. 2909-2914, 1996.

[6] Auricchio, F., Di Loreto, M. \& Sacco, E., Finite element analysis of a stenotic artery revascularization through stent insertion. Computer Methods in Biomechanics and Biomedical Engineering, 4, pp. 249-263, 2001. doi:10.1080/10255840108908007

[7] Migliavacca, F., Petrini, L., Colombo, M. et al., Mechanical behavior of coronary stents investigated through the finite element method. Journal of Biomechanics, 35, pp. 803-811, 2002. doi:10.1016/S0021-9290(02)00033-7

[8] Petrini, L., Migliavacca, F., Dubini, G. \& Auricchio, F., Evaluation of intravascular stent flexibility by means of numerical analysis. Proceedings of the 2003 Summer Bioengineering Conference, Key Biscayne: FL, pp. 251-252, 2003. 
[9] Lally, C., Dolan, F. \& Prendergast, P.J., Cardiovascular stent design and vessel stresses: a finite element analysis. Journal of Biomechanics, 38, pp. 1574-1581, 2005. doi:10.1016/j. jbiomech.2004.07.022

[10] Lally, C. \& Prendergast, P.J., An investigation into the applicability of a Mooney-Rivlin constitutive equation for modeling vascular tissue in cardiovascular stenting procedures. Proceedings of the International Congress on Computational Biomechanics, Zaragoza: Spain, pp. 542-550, 2003.

[11] Prendergast, P.J., Lally, C., Daly, S., Reid, A.J., Lee, T.C., Quinn, D. \& Dolan, F., Analysis of prolapse in cardiovascular stents: a constitutive equation for vascular tissue and finite element modelling. ASME Journal of Biomechanical Engineering, 125, pp. 692-699, 2003. doi:10.1115/1.1613674

[12] Loree, H.M., Grodzinsky, A.J., Park, S.Y., Gibson, L.J. \& Lee, R.T., Static circumferential tangential modulus of human artherosclerotic tissue. Journal of Biomechanics, 27, pp. 195-204, 1994. doi:10.1016/0021-9290(94)90209-7

[13] McFadden, E.P., Stabile, E., Regar, E., Cheneau, E. et al., Late thrombosis in drug-eluting coronary stents after discontinuation of antiplatelet therapy. The Lancet, 364, pp. 1519-1521, 2004. doi:10.1016/S0140-6736(04)17275-9

[14] Nichols, W.W. \& O'Rourke, M.F., McDonald's Blood Flow in Arteries Theoretical, Experimental and Clinical Principles, Oxford University Press: New York, 1998.

[15] Kuchulakanti, P.K., Chu, W.W., Torguson, R., Ohlmann, P. et al., Correlates and long-term outcomes of angiographically proven stent thrombosis with sirolimus- and paclitaxel-eluting stents. Circulation, 113, pp. 1108-1113, 2006. doi:10.1161/CIRCULATIONAHA.105.600155

[16] Ong, A.T., McFadden, E.P., Regar, E., de Jaegere, P.P., van Domburg, R.T. \& Serruys, P.W., Late angiographic stent thrombosis events with drug eluting stents. Journal of the American College of Cardiology, 45, pp. 2088-2092, 2005. doi:10.1016/j.jacc.2005.02.086 